\title{
含噻唑烷二酮-3-乙酸结构查尔酮衍生物的合成及抗菌活性的研究
}

\author{
孟凡领郑昌吉李因晶孙良鹏刘学坤 \\ 张天一朴虎日* \\ (延边大学药学院 长白山生物资源与功能分子教育部重点实验室 延吉 133002)
}

\begin{abstract}
摘要 合成了一系列含噻唑烷二酮-3-乙酸结构的新型查尔酮衍生物, 并对化合物进行了抗菌活性测定. 结果显示, 一 些化合物对 4 种多重耐药菌显示出较强的抗菌活性, 其中化合物 $8 \mathrm{~g}, 8 \mathrm{i}, 8 \mathrm{8}$ 和 $8 \mathrm{~m}$ 在抗耐甲氧西林金黄色葡萄球菌的最 小抑制浓度(MIC)达到 $4 \mu \mathrm{g} / \mathrm{mL}$, 与对照药诺氟沙星(norfloxacin)相当. 另外, 在 $64 \mu \mathrm{g} / \mathrm{mL}$ 浓度下, 所有化合物对大肠杆 菌 1356 均无明显抑制活性.

关键词 查尔酮; 噻唑烷二酮-3-乙酸; 抗菌活性
\end{abstract}

\section{Synthesis and Anti-bacterial Activity of Novel Chalcone Derivatives Containing 2,4-Thiazolidinedione-3-acetic Acid Moiety}

\author{
Meng, Fanling Zheng, Changji Li, Yinjing Sun, Liangpeng \\ Liu, Xuekun Zhang, Tianyi Piao, Huri* \\ (Key Laboratory of Natural Resources of Changbai Mountain \& Functional Molecules, Ministry of Education, \\ College of Pharmacy, Yanbian University, Yanji 133002)
}

\begin{abstract}
A series of novel chalcone derivatives bearing the 2,4-thiazolidinedione-3-acetic acid moieties $(\mathbf{8 a} \sim \mathbf{8 p})$ were synthesized, and evaluated for their anti-bacterial activity. The results demonstrated that some compounds presented good antimicrobial activities against four multidrug-resistant clinical isolates, among which compounds $\mathbf{8 g}, \mathbf{8 i}$, $8 \mathbf{1}$ and $\mathbf{8 m}$ with minimum inhibitory concentration (MIC) value of $4 \mu \mathrm{g} / \mathrm{mL}$ showed as active as the standard drug, norfloxacin, against methicillin-resistant $S$. aureus. None of the compounds exhibited obvious activity against the Gram-negative bacteria E. coli 1356 at $64 \mu \mathrm{g} / \mathrm{mL}$.

Keywords chalcone; 2,4-thiazolidinedione-3-acetic acid; anti-bacterial activity
\end{abstract}

目前, 随着抗菌药物的广泛使用甚至滥用, 细菌耐 药性也逐年增加, 耐药性细菌感染严重危害人类健康. 因此, 研发能够有效对付革兰阳性菌特别是耐药型细菌 所引起感染新型抗菌药已迫在眉睫 ${ }^{[1]}$.

查尔酮，其基本骨架结构为 1,3-二芳基丙烯酮，广 泛存在于天然植物中. 查尔酮类化合物分子具有较大的 柔性且能与不同的受体结合, 使其本身具有重要的药理 作用, 包括抗炎 ${ }^{[2]}$ 、抗真菌 ${ }^{[3]}$ 、抗肿瘤 ${ }^{[4]}$ 、抗菌 ${ }^{[5]}$ 等多种 生物活性. 此外, 噻唑烷二酮结构也具有优良的生物活 性 $^{[6]}$, 其环上的羰基及 $\mathrm{N}$ 原子可以参与生物体中氢键的 形成, 增加药物与受体的亲和力和选择性 ${ }^{[7]}$, 也是抗糖 尿病药物的重要片段. 近来研究发现, 噻唑烷二醞-3-乙
酸衍生物具有较强的抗菌活性 ${ }^{[8]}$.

基于以上研究, 依据生物活性叠加原理, 将查尔酮 与噻唑烷二酮两部分拼合，以期发现抗菌活性更好、毒 性低、抗菌谱广的化合物. 本研究合成了 16 个未见报道 的化合物, 目标化合物经 IR, ${ }^{1} \mathrm{H}$ NMR, MS 和元素分析 测试确认. 抗菌活性测试结果表明, 部分化合物具有较 好的抗菌活性. 合成路线如 Scheme 1 所示.

\section{1 实验部分}

\section{1 仪器和试剂}

熔点用毛细管法测定，温度计未经校正；IR 用

*E-mail: piaohuri@ybu.edu.cn

Received July 18, 2011; accepted September 14, 2011; accepted September 14, 2011.

Project supported by the National Natural Science Foundation of China (No. 20962021).

国家自然科学基金(No. 20962021)资助项目. 

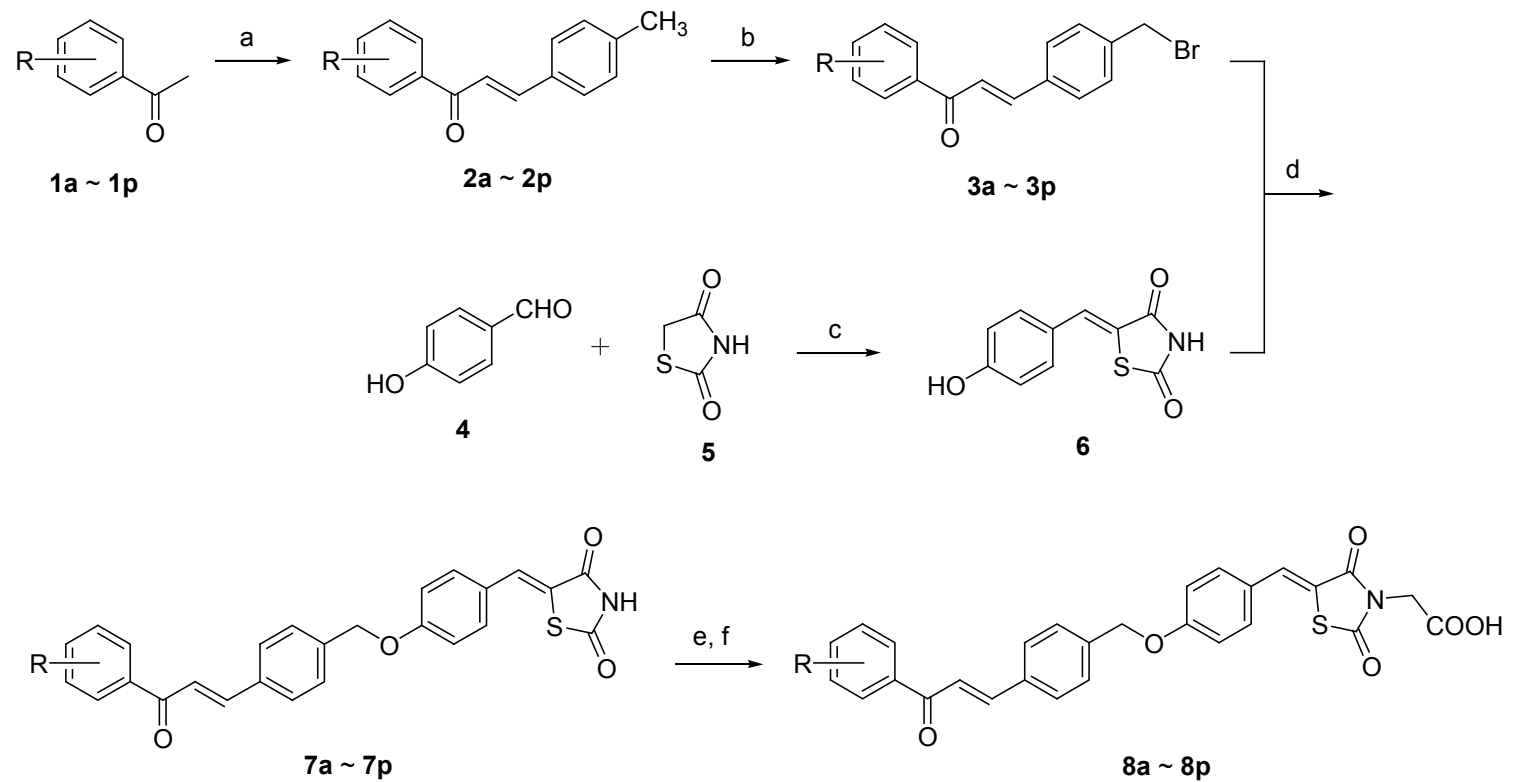

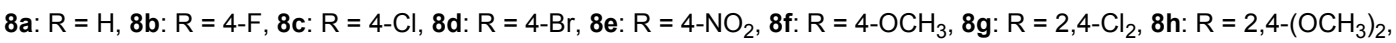

8i: $\mathrm{R}=2,4,6-\left(\mathrm{OCH}_{3}\right)_{3}, \mathbf{8 j}: \mathrm{R}=2-\mathrm{F}, \mathbf{8 k}: \mathrm{R}=2-\mathrm{Cl}, \mathbf{8 I}: \mathrm{R}=2-\mathrm{Br}, \mathbf{8 m}: \mathrm{R}=2-\mathrm{OCH}_{3}, \mathbf{8 n}: \mathrm{R}=3-\mathrm{Cl}, \mathbf{8 0}: \mathrm{R}=3-\mathrm{Br}, \mathbf{8 p}: \mathrm{R}=3-\mathrm{OCH}$

Reagents and conditions: (a) 4-methylbenzaldehyde, $\mathrm{EtOH} / \mathrm{H}_{2} \mathrm{O}$, $\mathrm{KOH}$, r.t.; (b) NBS, $\mathrm{CCl}_{4}, \mathrm{AlBN}, \mathrm{hv}, 2 \mathrm{~h}$; (c) $\mathrm{EtOH}$, piperidine/CH $\mathrm{COOH}_{3}$ reflux, $4 \mathrm{~h}$; (d) $\mathrm{K}_{2} \mathrm{CO}_{3}$, acetone, reflux, $4 \mathrm{~h}$; (e) $\mathrm{BrCH}_{2} \mathrm{CO}_{2} \mathrm{Bu}-t, \mathrm{~K}_{2} \mathrm{CO}_{3}$, acetone, reflux, $2 \mathrm{~h}$; (f) $\mathrm{CF}_{3} \mathrm{COOH}, \mathrm{CH}_{2} \mathrm{Cl}_{2}$, r.t., $3 \mathrm{~h}$

\section{Scheme 1}

FI-IR1730 型红外分光光度计测定( $\mathrm{KBr}$ 压片); ${ }^{1} \mathrm{H}$ NMR 用 BRUKER AV-300 型核磁共振仪测定(TMS 为内标, $\mathrm{CDCl}_{3}$ 或 DMSO- $d_{6}$ 为 溶 剂); ESI-MS 用 Agilent $1100 \mathrm{LC} / \mathrm{DAD} / \mathrm{MSD}$ 型液相色谱质谱联用仪; 元素分析 仪为 Perkin-Elmer 204Q 型; 酶标仪为 Bio-RAD 680 型. 本实验所用试剂均为 $\mathrm{AR}$ 级或 $\mathrm{CP}$ 级.

\section{2 化合物的合成}

1.2.1 (E)-1-取代苯基-3-(4-甲基苯基)-2-丙烯-1-酮 $(2 a \sim 2 p)$ 的合成

查尔酮 2a 2p 由不同取代的苯乙酮与对甲基苯甲 醛在乙醇/碱液体系中室温反应得到 ${ }^{[9]}$.

1.2.2（E)-3-(4-溴甲基苯基)-1-取代苯基-2-丙烯-1-酮 $(3 \mathbf{a} \sim 3 p)$ 的合成

在 $25 \mathrm{~mL}$ 单口圆底烧瓶中加入 $1 \mathrm{mmol}$ 的查尔酮衍 生物 $2,1.3 \mathrm{mmol}$ 的 $N$-溴代琥珀酰亚胺(NBS), $10 \mathrm{~mL}$ 新 蒸的 $\mathrm{CCl}_{4}$, 用偶氮二异丁睛(AIBN)作引发剂, 置光照下 反应 $2 \mathrm{~h}$, 冷却至室温, 减压蒸除溶剂. 然后用乙醇洗 涤, 抽滤, 得白色粉末状固体 $\mathbf{3 a} \sim \mathbf{3 p}$, 用于下一步.

化合物 3c: 产率 93\%. m.p. $160 \sim 162{ }^{\circ} \mathrm{C} ;{ }^{1} \mathrm{H}$ NMR $\left(\mathrm{CDCl}_{3}, 300 \mathrm{MHz}\right) \delta: 4.72\left(\mathrm{~s}, 2 \mathrm{H}, \mathrm{CH}_{2}\right), 7.60(\mathrm{~d}, J=15.6$ $\mathrm{Hz}, 1 \mathrm{H}, \mathrm{CH}=\mathrm{CH}), 7.92(\mathrm{~d}, J=15.6 \mathrm{~Hz}, 1 \mathrm{H}, \mathrm{CH}=\mathrm{CH})$, $7.01 \sim 8.06(\mathrm{~m}, 8 \mathrm{H}, \mathrm{ArH})$.
1.2.3 化合物 (Z)-5-(4-着基苄亚甲基)-2,4-噻唑烷二 酮(6)的合成

将 $12 \mathrm{mmol}$ 的对羟基苯甲醛(4), $10 \mathrm{mmol}$ 的 2,4-噻 唑烷二酮(5)加入到 $25 \mathrm{~mL}$ 无水乙醇中, 然后加入无水哌 啶和冰醋酸各 5 滴，回流反应 $4 \mathrm{~h}$, 冷却至室温，将所得 沉淀抽滤得深黄色固体 $1.98 \mathrm{~g}$, 产率 90\%, m.p. 302 $304{ }^{\circ} \mathrm{C}$ (文献值 ${ }^{[10]}$ : m.p. $301 \sim 303{ }^{\circ} \mathrm{C}$ ).

1.2.4 (Z)-5-\{4-[4-((E)-3-氧代-3-取代苯基-1-丙烯基) 華甲氧基]芐亚甲基 $\}-2,4$-噻唑烷二酮 $(7 \mathbf{a} \sim 7 \mathbf{p}$ ) 的合成

在装有 $10 \mathrm{~mL}$ 干燥丙酮的 $25 \mathrm{~mL}$ 的圆底烧瓶中, 加 入 $1 \mathrm{mmol}$ 中间体 3,1 $\mathrm{mmol}$ 的化合物 $\mathbf{6}$ 及 $1.5 \mathrm{mmol}$ 的 碳酸钾, 回流反应 $4 \mathrm{~h}$, 冷却至室温, 减压蒸除溶剂. 然 后加入适量乙酸乙酯，摚拌使其溶解，用饱和食盐水洗 涤两次，无水硫酸镁干燥过夜，过滤，回收溶剂后得到 粗品. 经柱层析 $[V$ (二氯甲烷 $): V$ (甲醇 $)=60 ： 1]$ 得到淡 黄色固体 7a $\sim 7 \mathbf{p}$. 其中化合物 7a 的产率、熔点及波谱 数据如下: 产率 $82 \%$. m.p. $150 \sim 152{ }^{\circ} \mathrm{C} ;{ }^{1} \mathrm{H}$ NMR $\left(\right.$ DMSO- $\left.d_{6}, 300 \mathrm{MHz}\right) \delta: 4.88\left(\mathrm{~s}, 2 \mathrm{H}, \mathrm{CH}_{2}\right), 7.72(\mathrm{~d}, J=$ $15.3 \mathrm{~Hz}, 1 \mathrm{H}, \mathrm{CH}=\mathrm{CH}), 7.89(\mathrm{~s}, 1 \mathrm{H}, \mathrm{CH}), 7.91(\mathrm{~d}, J=15.3$ $\mathrm{Hz}, 1 \mathrm{H}, \mathrm{CH}=\mathrm{CH}), 6.92 \sim 8.15(\mathrm{~m}, 13 \mathrm{H}, \mathrm{ArH}), 10.37(\mathrm{~s}$, $1 \mathrm{H}, \mathrm{NH})$; IR (KBr) $v$ : $3306(\mathrm{NH}), 1736(\mathrm{C}=\mathrm{O}), 1668(\mathrm{C}=$ O), $1598(\mathrm{C}=\mathrm{C}) 1595(\mathrm{C}=\mathrm{C}) \mathrm{cm}^{-1}$; MS m/z: $442([\mathrm{M}+$ 1] ${ }^{+}$). Anal. calcd for $\mathrm{C}_{26} \mathrm{H}_{19} \mathrm{NO}_{4} \mathrm{~S}: \mathrm{C} 70.73, \mathrm{H} 4.34, \mathrm{~N} 3.17$, 
S 7.26; found C 70.81, H 4.13, N 3.28, S 7.31.

$1.2 .52-[(Z)-5-\{4-[4-((E)-3-$ 氧代-3-取代苯基- 1-丙烯 基)芐甲氧基]芐亚甲基\}-2,4-噻唑烷二酮-3-基]乙酸 $(8 \mathrm{a} \sim 8 \mathrm{p})$ 的合成

$25 \mathrm{~mL}$ 的圆底烧瓶中, 加入 $1 \mathrm{mmol}$ 化合物 7, 1.5 $\mathrm{mmol}$ 的无水碳酸钾和干燥丙酮 $10 \mathrm{~mL}$, 回流 $0.5 \mathrm{~h}$. 冷 却至室温, 滴加 $2 \mathrm{mmol}$ 溴乙酸叔丁酯, $50{ }^{\circ} \mathrm{C}$ 下搅拌 2 $h$, 冷却至室温, 减压蒸除溶剂. 然后加入适量乙酸乙 酯, 摚拌使其溶解, 用饱和食盐水洗涤两次, 无水硫酸 镁干燥过夜, 抽滤, 回收溶剂后得到无色油状物. 向装 有油状物的圆底烧瓶中加入含 $20 \%$ 三氟乙酸的二氯甲 烷溶液 $(V: V=1: 4)$, 室温反应 $3 \mathrm{~h}$ 后, 减压蒸除溶剂 至瓶底有少量液体时, 加入 $5 \mathrm{~mL}$ 水, 产生白色沉淀, 抽 滤, 水洗滤饼, 干燥, 然后再用少量乙醚洗涤, 抽滤, 干 燥得白色固体 $8 \mathrm{a} \sim 8 \mathrm{p}$. 化合物 $8 \mathrm{a} \sim 8 \mathrm{p}$ 的产率、熔点及 波谱数据如下:

8a: 产率 88\%. m.p. $198 \sim 200{ }^{\circ} \mathrm{C} ;{ }^{1} \mathrm{H}$ NMR (DMSO$\left.d_{6}, 300 \mathrm{MHz}\right) \delta: 4.77\left(\mathrm{~s}, 2 \mathrm{H}, \mathrm{CH}_{2}\right), 4.87\left(\mathrm{~s}, 2 \mathrm{H}, \mathrm{CH}_{2}\right), 7.69$ $(\mathrm{d}, J=15.6 \mathrm{~Hz}, 1 \mathrm{H}, \mathrm{CH}=\mathrm{CH}), 7.89(\mathrm{~d}, J=15.6 \mathrm{~Hz}, 1 \mathrm{H}$, $\mathrm{CH}=\mathrm{CH}), 7.92(\mathrm{~s}, 1 \mathrm{H}, \mathrm{CH}), 7.06 \sim 8.13(\mathrm{~m}, 13 \mathrm{H}, \mathrm{ArH})$, $13.08(\mathrm{~s}, 1 \mathrm{H}, \mathrm{COOH})$; IR (KBr) v: $3053(\mathrm{OH}), 1736(\mathrm{C}=$ O), $1678(\mathrm{C}=\mathrm{O}), 1595(\mathrm{C}=\mathrm{C}) \mathrm{cm}^{-1}$; MS m/z: 500 ([M+ 1] ${ }^{+}$). Anal. calcd for $\mathrm{C}_{28} \mathrm{H}_{21} \mathrm{NO}_{6} \mathrm{~S}: \mathrm{C} 67.32, \mathrm{H} 4.24, \mathrm{~N} 2.80$, S 6.42; found C 67.43, H 4.32, N 2.68, S 6.35.

8b: 产率 86\%. m.p. 208 $210{ }^{\circ} \mathrm{C}$; ${ }^{1} \mathrm{H}$ NMR (DMSO$\left.d_{6}, 300 \mathrm{MHz}\right) \delta: 4.76\left(\mathrm{~s}, 2 \mathrm{H}, \mathrm{CH}_{2}\right), 4.86\left(\mathrm{~s}, 2 \mathrm{H}, \mathrm{CH}_{2}\right), 7.69$ $(\mathrm{d}, J=15.6 \mathrm{~Hz}, 1 \mathrm{H}, \mathrm{CH}=\mathrm{CH}), 7.90(\mathrm{~d}, J=15.6 \mathrm{~Hz}, 1 \mathrm{H}$, $\mathrm{CH}=\mathrm{CH}), 7.92(\mathrm{~s}, 1 \mathrm{H}, \mathrm{CH}), 7.06 \sim 8.24(\mathrm{~m}, 12 \mathrm{H}, \mathrm{ArH})$, 13.15 (s, 1H, COOH); IR (KBr) v: $3060(\mathrm{OH}), 1736(\mathrm{C}=$ O), $1680(\mathrm{C}=\mathrm{O}), 1596(\mathrm{C}=\mathrm{C}) \mathrm{cm}^{-1}$; MS m/z: 518 ([M+ 1] ${ }^{+}$). Anal. calcd for $\mathrm{C}_{28} \mathrm{H}_{20} \mathrm{FNO}_{6} \mathrm{~S}: \mathrm{C}$ 64.98, $\mathrm{H}$ 3.90, N 2.71, S 6.20; found C 64.92, H 4.13, N 2.83, S 6.15.

8c: 产率 86\%. m.p. 252 254 ${ }^{\circ} \mathrm{C}$; ${ }^{1} \mathrm{H}$ NMR (DMSO$\left.d_{6}, 300 \mathrm{MHz}\right) \delta: 4.76\left(\mathrm{~s}, 2 \mathrm{H}, \mathrm{CH}_{2}\right), 4.86\left(\mathrm{~s}, 2 \mathrm{H}, \mathrm{CH}_{2}\right), 7.70$ $(\mathrm{d}, J=15.6 \mathrm{~Hz}, 1 \mathrm{H}, \mathrm{CH}=\mathrm{CH}), 7.88(\mathrm{~d}, J=15.6 \mathrm{~Hz}, 1 \mathrm{H}$, $\mathrm{CH}=\mathrm{CH}), 7.92(\mathrm{~s}, 1 \mathrm{H}, \mathrm{CH}), 7.06 \sim 8.15(\mathrm{~m}, 12 \mathrm{H}, \mathrm{ArH})$, 13.10 (s, 1H, COOH); IR (KBr) v: $3048(\mathrm{OH}), 1739(\mathrm{C}=$ O), $1682(\mathrm{C}=\mathrm{O}), 1597(\mathrm{C}=\mathrm{C}) \mathrm{cm}^{-1}$; MS m/z: $535([\mathrm{M}+$ 1] ${ }^{+}$). Anal. calcd for $\mathrm{C}_{28} \mathrm{H}_{20} \mathrm{ClNO}_{6} \mathrm{~S}$ : C 62.98, H 3.78, N 2.62, S 6.00; found C 62.92, H 3.91, N 2.55, S 5.93.

8d: 产率 84\%. m.p. $251 \sim 253{ }^{\circ} \mathrm{C} ;{ }^{1} \mathrm{H}$ NMR (DMSO$\left.d_{6}, 300 \mathrm{MHz}\right) \delta: 4.82\left(\mathrm{~s}, 2 \mathrm{H}, \mathrm{CH}_{2}\right), 4.91\left(\mathrm{~s}, 2 \mathrm{H}, \mathrm{CH}_{2}\right), 7.75$ $(\mathrm{d}, J=15.6 \mathrm{~Hz}, 1 \mathrm{H}, \mathrm{CH}=\mathrm{CH}), 7.93(\mathrm{~d}, J=15.6 \mathrm{~Hz}, 1 \mathrm{H}$, $\mathrm{CH}=\mathrm{CH}), 7.96(\mathrm{~s}, 1 \mathrm{H}, \mathrm{CH}), 7.10 \sim 8.12(\mathrm{~m}, 12 \mathrm{H}, \mathrm{ArH})$, $13.14(\mathrm{~s}, 1 \mathrm{H}, \mathrm{COOH})$; IR (KBr) v: $3058(\mathrm{OH}), 1735(\mathrm{C}=$
O), $1674(\mathrm{C}=\mathrm{O}), 1596(\mathrm{C}=\mathrm{C}) \mathrm{cm}^{-1}$; MS m/z 579 ([M+ $1]^{+}$). Anal. calcd for $\mathrm{C}_{28} \mathrm{H}_{20} \mathrm{BrNO}_{6} \mathrm{~S}$ : C 58.14, H 3.49, N 2.42, S 5.54; found C 58.01, H 3.42, N 2.56, S 5.63.

8e: 产率 92\%. m.p. $249 \sim 251{ }^{\circ} \mathrm{C} ;{ }^{1} \mathrm{H}$ NMR (DMSO$\left.d_{6}, 300 \mathrm{MHz}\right) \delta: 4.73\left(\mathrm{~s}, 2 \mathrm{H}, \mathrm{CH}_{2}\right), 4.87\left(\mathrm{~s}, 2 \mathrm{H}, \mathrm{CH}_{2}\right), 7.75$ $(\mathrm{d}, J=15.6 \mathrm{~Hz}, 1 \mathrm{H}, \mathrm{CH}=\mathrm{CH}), 7.90(\mathrm{~d}, J=15.6 \mathrm{~Hz}, 1 \mathrm{H}$, $\mathrm{CH}=\mathrm{CH}), 7.91(\mathrm{~s}, 1 \mathrm{H}, \mathrm{CH}), 7.06 \sim 8.37(\mathrm{~m}, 12 \mathrm{H}, \mathrm{ArH})$, 13.11 (s, 1H, COOH); IR (KBr) v: $3049(\mathrm{OH}), 1741(\mathrm{C}=$ O), $1683(\mathrm{C}=\mathrm{O}), 1598(\mathrm{C}=\mathrm{C}) \mathrm{cm}^{-1}$; MS m/z 545 ([M+ $1]^{+}$). Anal. calcd for $\mathrm{C}_{28} \mathrm{H}_{20} \mathrm{~N}_{2} \mathrm{O}_{8} \mathrm{~S}$ : C 61.76, H 3.70, N 5.14, S 5.89; found C 61.88, H 3.61, N 5.26, S 5.73.

8f: 产率 $86 \%$. m.p. $238 \sim 240{ }^{\circ} \mathrm{C} ;{ }^{1} \mathrm{H}$ NMR (DMSO$\left.d_{6}, 300 \mathrm{MHz}\right) \delta: 3.85\left(\mathrm{~s}, 3 \mathrm{H}, \mathrm{OCH}_{3}\right), 4.77\left(\mathrm{~s}, 2 \mathrm{H}, \mathrm{CH}_{2}\right)$, $4.86\left(\mathrm{~s}, 2 \mathrm{H}, \mathrm{CH}_{2}\right), 7.65(\mathrm{~d}, J=15.6 \mathrm{~Hz}, 1 \mathrm{H}, \mathrm{CH}=\mathrm{CH})$, $7.84(\mathrm{~d}, J=15.6 \mathrm{~Hz}, 1 \mathrm{H}, \mathrm{CH}=\mathrm{CH}), 7.92(\mathrm{~s}, 1 \mathrm{H}, \mathrm{CH})$, $7.05 \sim 8.14(\mathrm{~m}, 12 \mathrm{H}, \mathrm{ArH}), 13.09(\mathrm{~s}, 1 \mathrm{H}, \mathrm{COOH}) ; \mathrm{IR}$ (KBr) v: $3062(\mathrm{OH}), 1734(\mathrm{C}=\mathrm{O}), 1678(\mathrm{C}=\mathrm{O}), 1593$ $(\mathrm{C}=\mathrm{C}) \mathrm{cm}^{-1}$; MS m/z $530\left([\mathrm{M}+1]^{+}\right)$. Anal. calcd for $\mathrm{C}_{29} \mathrm{H}_{23} \mathrm{NO}_{7} \mathrm{~S}$ : C 65.77, $\mathrm{H}$ 4.38; N 2.64, S 6.06; found $\mathrm{C}$ 65.83, H 4.32, N 2.78; S 5.99.

8g: 产率 82\%. m.p. $224 \sim 226{ }^{\circ} \mathrm{C} ;{ }^{1} \mathrm{H}$ NMR (DMSO$\left.d_{6}, 300 \mathrm{MHz}\right) \delta: 4.79\left(\mathrm{~s}, 2 \mathrm{H}, \mathrm{CH}_{2}\right), 4.87\left(\mathrm{~s}, 2 \mathrm{H}, \mathrm{CH}_{2}\right), 7.24$ $(\mathrm{d}, J=15.6 \mathrm{~Hz}, 1 \mathrm{H}, \mathrm{CH}=\mathrm{CH}), 7.42(\mathrm{~d}, J=15.6 \mathrm{~Hz}, 1 \mathrm{H}$, $\mathrm{CH}=\mathrm{CH}), 7.08 \sim 7.79(\mathrm{~m}, 11 \mathrm{H}, \mathrm{ArH}), 7.93(\mathrm{~s}, 1 \mathrm{H}, \mathrm{CH})$, 13.17 (s, 1H, COOH); IR (KBr) v: $3051(\mathrm{OH}), 1739(\mathrm{C}=$ O), $1685(\mathrm{C}=\mathrm{O}), 1597(\mathrm{C}=\mathrm{C}) \mathrm{cm}^{-1}$; MS m/z 569 ([M+ $1]^{+}$). Anal. calcd for $\mathrm{C}_{28} \mathrm{H}_{19} \mathrm{Cl}_{2} \mathrm{NO}_{6} \mathrm{~S}$ : C 59.16, $\mathrm{H} 3.37, \mathrm{~N}$ 2.46, S 5.64; found C 59.01, H 3.28, N 2.59, S 5.68.

8h: 产率 91\%. m.p. $206 \sim 208{ }^{\circ} \mathrm{C} ;{ }^{1} \mathrm{H}$ NMR (DMSO$\left.d_{6}, 300 \mathrm{MHz}\right) \delta: 3.83\left(\mathrm{~s}, 3 \mathrm{H}, \mathrm{OCH}_{3}\right), 3.87\left(\mathrm{~s}, 3 \mathrm{H}, \mathrm{OCH}_{3}\right)$, 4.77 (s, 2H, $\left.\mathrm{CH}_{2}\right), 4.85$ (s, 2H, $\left.\mathrm{CH}_{2}\right), 7.24(\mathrm{~d}, J=15.6 \mathrm{~Hz}$, $1 \mathrm{H}, \mathrm{CH}=\mathrm{CH}), 7.53(\mathrm{~d}, J=15.6 \mathrm{~Hz}, 1 \mathrm{H}, \mathrm{CH}=\mathrm{CH})$, $6.60 \sim 6.69(\mathrm{~m}, 11 \mathrm{H}, \mathrm{ArH}), 7.91(\mathrm{~s}, 1 \mathrm{H}, \mathrm{CH}), 13.08(\mathrm{~s}, 1 \mathrm{H}$, $\mathrm{COOH})$; IR (KBr) v: $3065(\mathrm{OH}), 1737(\mathrm{C}=\mathrm{O}), 1674(\mathrm{C}=$ O), $1592(\mathrm{C}=\mathrm{C}) \mathrm{cm}^{-1}$; MS m/z $560\left([\mathrm{M}+1]^{+}\right)$. Anal. calcd for $\mathrm{C}_{30} \mathrm{H}_{25} \mathrm{NO}_{8} \mathrm{~S}$ : C 64.39, $\mathrm{H}$ 4.50, N 2.50, S 5.73; found C 64.26, H 4.43, N 2.44, S 5.87.

8i: 产率 89\%. m.p. $212 \sim 214{ }^{\circ} \mathrm{C} ;{ }^{1} \mathrm{H}$ NMR (DMSO$\left.d_{6}, 300 \mathrm{MHz}\right) \delta: 3.70\left(\mathrm{~s}, 6 \mathrm{H}, 2 \times \mathrm{OCH}_{3}\right), 3.83(\mathrm{~s}, 3 \mathrm{H}$, $\left.\mathrm{OCH}_{3}\right), 4.79\left(\mathrm{~s}, 2 \mathrm{H}, \mathrm{CH}_{2}\right), 4.85\left(\mathrm{~s}, 2 \mathrm{H}, \mathrm{CH}_{2}\right), 6.94$ (d, J= $15.9 \mathrm{~Hz}, 1 \mathrm{H}, \mathrm{CH}=\mathrm{CH}), 7.18(\mathrm{~d}, J=15.9 \mathrm{~Hz}, 1 \mathrm{H}, \mathrm{CH}=$ $\mathrm{CH}), 6.31 \sim 7.67$ (m, 10H, ArH), 7.93 (s, 1H, CH), 13.08 $(\mathrm{s}, 1 \mathrm{H}, \mathrm{COOH})$; IR $(\mathrm{KBr}) v: 3055(\mathrm{OH}), 1738(\mathrm{C}=\mathrm{O})$, $1681(\mathrm{C}=\mathrm{O}), 1594(\mathrm{C}=\mathrm{C}) \mathrm{cm}^{-1}$; $\mathrm{MS} \mathrm{m} / z 590\left([\mathrm{M}+1]^{+}\right)$. Anal. calcd for $\mathrm{C}_{31} \mathrm{H}_{27} \mathrm{NO}_{9} \mathrm{~S}$ : C 63.15, H 4.62, N 2.38, S 
5.44; found C 63.23, H 4.57, N 2.27, S 5.16.

8j: 产率 84\%. m.p. $198 \sim 200{ }^{\circ} \mathrm{C} ;{ }^{1} \mathrm{H}$ NMR (DMSO$\left.d_{6}, 300 \mathrm{MHz}\right) \delta: 4.78\left(\mathrm{~s}, 2 \mathrm{H}, \mathrm{CH}_{2}\right), 4.86\left(\mathrm{~s}, 2 \mathrm{H}, \mathrm{CH}_{2}\right), 7.25$ $(\mathrm{d}, J=16.2 \mathrm{~Hz}, 1 \mathrm{H}, \mathrm{CH}=\mathrm{CH}), 7.53(\mathrm{~d}, J=16.2 \mathrm{~Hz}, 1 \mathrm{H}$, $\mathrm{CH}=\mathrm{CH}), 6.96 \sim 7.76(\mathrm{~m}, 12 \mathrm{H}, \mathrm{ArH}), 7.93(\mathrm{~s}, 1 \mathrm{H}, \mathrm{CH})$, 13.13 (s, 1H, COOH); IR (KBr) v: $3060(\mathrm{OH}), 1736(\mathrm{C}=$ $\mathrm{O}), 1680(\mathrm{C}=\mathrm{O}), 1596(\mathrm{C}=\mathrm{C}) \mathrm{cm}^{-1}$; MS m/z $518([\mathrm{M}+$ 1] ${ }^{+}$). Anal. calcd for $\mathrm{C}_{28} \mathrm{H}_{20} \mathrm{FNO}_{6} \mathrm{~S}: \mathrm{C}$ 64.98, H 3.90, N 2.71, S 6.20; found C 64.88, H 4.12, N 2.79, S 6.17.

8k: 产率 88\%. m.p. 194 $196{ }^{\circ} \mathrm{C}$; ${ }^{1} \mathrm{H}$ NMR (DMSO$\left.d_{6}, 300 \mathrm{MHz}\right) \delta: 4.79\left(\mathrm{~s}, 2 \mathrm{H}, \mathrm{CH}_{2}\right), 4.87\left(\mathrm{~s}, 2 \mathrm{H}, \mathrm{CH}_{2}\right), 7.26$ (d, $J=15.6 \mathrm{~Hz}, 1 \mathrm{H}, \mathrm{CH}=\mathrm{CH}), 7.59$ (d, $J=15.6 \mathrm{~Hz}, 1 \mathrm{H}$, $\mathrm{CH}=\mathrm{CH}), 7.01 \sim 7.77(\mathrm{~m}, 12 \mathrm{H}, \mathrm{ArH}), 8.07(\mathrm{~s}, 1 \mathrm{H}, \mathrm{CH})$, 13.14 (s, 1H, COOH); IR (KBr) v: $3048(\mathrm{OH}), 1739(\mathrm{C}=$ O), $1682(\mathrm{C}=\mathrm{O}), 1597(\mathrm{C}=\mathrm{C}) \mathrm{cm}^{-1}$; MS m/z 535 ([M+ 1] ${ }^{+}$). Anal. calcd for $\mathrm{C}_{28} \mathrm{H}_{20} \mathrm{ClNO}_{6} \mathrm{~S}: \mathrm{C}$ 62.98, $\mathrm{H} \mathrm{3.78,} \mathrm{N}$ 2.62, S 6.00; found C 62.86, H 3.82, N 2.53, S 5.97.

81: 产率 90\%. m.p. 208 210 ${ }^{\circ} \mathrm{C} ;{ }^{1} \mathrm{H}$ NMR (DMSO$\left.d_{6}, 300 \mathrm{MHz}\right) \delta: 4.79\left(\mathrm{~s}, 2 \mathrm{H}, \mathrm{CH}_{2}\right), 4.89\left(\mathrm{~s}, 2 \mathrm{H}, \mathrm{CH}_{2}\right), 7.75$ $(\mathrm{d}, J=15.6 \mathrm{~Hz}, 1 \mathrm{H}, \mathrm{CH}=\mathrm{CH}), 7.97(\mathrm{~d}, J=15.6 \mathrm{~Hz}, 1 \mathrm{H}$, $\mathrm{CH}=\mathrm{CH}), 7.09 \sim 8.16(\mathrm{~m}, 12 \mathrm{H}, \mathrm{ArH}), 7.31(\mathrm{~s}, 1 \mathrm{H}, \mathrm{CH})$, 13.13 (s, 1H, COOH); IR (KBr) v: $3058(\mathrm{OH}), 1735(\mathrm{C}=$ $\mathrm{O}), 1674(\mathrm{C}=\mathrm{O}), 1596(\mathrm{C}=\mathrm{C}) \mathrm{cm}^{-1}$; MS m/z $579([\mathrm{M}+$ $1]^{+}$). Anal. calcd for $\mathrm{C}_{28} \mathrm{H}_{20} \mathrm{BrNO}_{6} \mathrm{~S}$ : C 58.14, H 3.49, N 2.42, S 5.54; found C 58.05, H 3.52, N 2.47, S 5.67.

8m: 产率 87\%. m.p. $192 \sim 194{ }^{\circ} \mathrm{C} ;{ }^{1} \mathrm{H}$ NMR $\left(\mathrm{DMSO}-d_{6}, 300 \mathrm{MHz}\right) \delta: 3.86\left(\mathrm{~s}, 3 \mathrm{H}, \mathrm{OCH}_{3}\right), 4.80(\mathrm{~s}, 2 \mathrm{H}$, $\left.\mathrm{CH}_{2}\right), 4.87\left(\mathrm{~s}, 2 \mathrm{H}, \mathrm{CH}_{2}\right), 7.20(\mathrm{~d}, J=15.9 \mathrm{~Hz}, 1 \mathrm{H}, \mathrm{CH}=$ $\mathrm{CH}), 7.55(\mathrm{~d}, J=15.9 \mathrm{~Hz}, 1 \mathrm{H}, \mathrm{CH}=\mathrm{CH}), 7.04 \sim 7.74(\mathrm{~m}$, 12H, ArH), 7.94 (s, 1H, CH), 13.14 (s, 1H, COOH); IR $(\mathrm{KBr}) v: 3062(\mathrm{OH}), 1734(\mathrm{C}=\mathrm{O}), 1678(\mathrm{C}=\mathrm{O}), 1593$ $(\mathrm{C}=\mathrm{C}) \mathrm{cm}^{-1}$; MS m/z $530\left([\mathrm{M}+1]^{+}\right)$. Anal. calcd for $\mathrm{C}_{29} \mathrm{H}_{23} \mathrm{NO}_{7} \mathrm{~S}$ : C 65.77, $\mathrm{H}$ 4.38; N 2.64, $\mathrm{S}$ 6.06; found $\mathrm{C}$ 65.80, H 4.42, N 2.73; S 5.95.

8n: 产率 93\%. m.p. $220 \sim 222{ }^{\circ} \mathrm{C} ;{ }^{1} \mathrm{H}$ NMR (DMSO$\left.d_{6}, 300 \mathrm{MHz}\right) \delta: 4.80\left(\mathrm{~s}, 2 \mathrm{H}, \mathrm{CH}_{2}\right), 4.89\left(\mathrm{~s}, 2 \mathrm{H}, \mathrm{CH}_{2}\right), 7.54$ $(\mathrm{d}, J=15.6 \mathrm{~Hz}, 1 \mathrm{H}, \mathrm{CH}=\mathrm{CH}), 7.95(\mathrm{~d}, J=15.6 \mathrm{~Hz}, 1 \mathrm{H}$, $\mathrm{CH}=\mathrm{CH}), 7.10 \sim 8.12(\mathrm{~m}, 12 \mathrm{H}, \mathrm{ArH}), 8.19(\mathrm{~s}, 1 \mathrm{H}, \mathrm{CH})$, 13.13 (s, 1H, COOH); IR (KBr) v: $3048(\mathrm{OH}), 1739(\mathrm{C}=$ O), $1682(\mathrm{C}=\mathrm{O}), 1597(\mathrm{C}=\mathrm{C}) \mathrm{cm}^{-1}$; MS m/z 535 ([M+ $\left.1]^{+}\right)$. Anal. calcd for $\mathrm{C}_{28} \mathrm{H}_{20} \mathrm{ClNO}_{6} \mathrm{~S}$ : C 62.98, H 3.78, N 2.62, S 6.00; found C 62.91, H 3.89, N 2.58, S 5.93.

8o: 产率 89\%. m.p. 226 228 ${ }^{\circ} \mathrm{C} ;{ }^{1} \mathrm{H}$ NMR (DMSO$\left.d_{6}, 300 \mathrm{MHz}\right) \delta: 4.78\left(\mathrm{~s}, 2 \mathrm{H}, \mathrm{CH}_{2}\right), 4.88\left(\mathrm{~s}, 2 \mathrm{H}, \mathrm{CH}_{2}\right), 7.73$ $(\mathrm{d}, J=15.6 \mathrm{~Hz}, 1 \mathrm{H}, \mathrm{CH}=\mathrm{CH}), 7.93(\mathrm{~d}, J=15.6 \mathrm{~Hz}, 1 \mathrm{H}$,
$\mathrm{CH}=\mathrm{CH}), 7.07 \sim 8.14(\mathrm{~m}, 12 \mathrm{H}, \mathrm{ArH}), 8.30(\mathrm{~s}, 1 \mathrm{H}, \mathrm{CH})$, $13.11(\mathrm{~s}, 1 \mathrm{H}, \mathrm{COOH})$; IR $(\mathrm{KBr}) v: 3058(\mathrm{OH}), 1735(\mathrm{C}=$ O), $1674(\mathrm{C}=\mathrm{O}), 1596(\mathrm{C}=\mathrm{C}) \mathrm{cm}^{-1}$; MS m/z 579 ([M+ $1]^{+}$). Anal. calcd for $\mathrm{C}_{28} \mathrm{H}_{20} \mathrm{BrNO}_{6} \mathrm{~S}$ : C 58.14, H 3.49, N 2.42, S 5.54; found C 58.13, H 3.47, N 2.50, S 5.57.

8p: 产率 95\%. m.p. 190 $192{ }^{\circ} \mathrm{C} ;{ }^{1} \mathrm{H}$ NMR (DMSO$\left.d_{6}, 300 \mathrm{MHz}\right) \delta: 3.85\left(\mathrm{~s}, 3 \mathrm{H}, \mathrm{OCH}_{3}\right), 4.79\left(\mathrm{~s}, 2 \mathrm{H}, \mathrm{CH}_{2}\right)$, $4.89\left(\mathrm{~s}, 2 \mathrm{H}, \mathrm{CH}_{2}\right), 7.72(\mathrm{~d}, J=15.6 \mathrm{~Hz}, 1 \mathrm{H}, \mathrm{CH}=\mathrm{CH})$, $7.86(\mathrm{~d}, J=15.6 \mathrm{~Hz}, 1 \mathrm{H}, \mathrm{CH}=\mathrm{CH}), 7.05 \sim 8.14(\mathrm{~m}, 12 \mathrm{H}$, $\mathrm{ArH}), 7.94$ (s, 1H, CH), 13.14 (s, 1H, COOH); IR (KBr) v: $3062(\mathrm{OH}), 1734(\mathrm{C}=\mathrm{O}), 1678(\mathrm{C}=\mathrm{O}), 1593(\mathrm{C}=\mathrm{C})$ $\mathrm{cm}^{-1}$; MS m/z $530\left([\mathrm{M}+1]^{+}\right)$. Anal. calcd for $\mathrm{C}_{29} \mathrm{H}_{23} \mathrm{~N}$ $\mathrm{O}_{7} \mathrm{~S}$ : C 65.77, H 4.38, N 2.64, S 6.06; found C 65.81, H $4.29, \mathrm{~N} 2.57$; S 6.11 .

\section{3 抗菌活性的测试}

所测菌株：金黄色葡萄球菌(S. aureus 4220)、变形 链球菌 $(S$. mutans 3056)、大肠杆菌 $(E$. coli 1356)、耐甲 氧西林金黄色葡萄球菌(methicillin-resistant $S$. aureus)和 耐喹诺酩金黄色葡萄球菌(quinolone-resistant $S$. aureus).

具体测试方法参照文献[11]，以诺氟沙星(norfloxacin)和苯唑西林(oxacillin)为阳性对照药物, 判定指标为 最小抑菌浓度(MIC， $\mu \mathrm{g} / \mathrm{mL})$. 在抗金黄色葡萄球菌初笁 结果基础上，选择抗菌活性较好的化合物 $8 \mathrm{~g}, 8 \mathrm{i}, 8 \mathrm{I}$ 和 $8 \mathrm{~m}$ 做了 4 种多重耐药菌的活性测定.

\section{2 结果与讨论}

\section{1 合成}

在溴化物 3a 3p 合成过程中，选择较温和的 NBS 进行溴代. 由于文献报道此类反应均没提及光线问 题 ${ }^{[12]}$, 所以我们开始在室内正常光线下进行反应，发现 底物不能完全反应，而且如果继续增加 NBS 的量，得到 的一溴中间体 $3 \mathbf{a} \sim \mathbf{3 p}$ 会继续溴化为二溴代产物，蒸除 溶剂后得到淡黄色粘稠物，借助柱层析才能纯化. 后来 在阳光下进行反应, NBS 能顺利溴代反应，当 NBS 加入 量为 1.3 equiv. 时, 无二溴化产物生成, 且反应时间明显 缩短，产率达 $90 \%$ 左右，蒸除反应液后得到白色固体， 经乙醇洗涤可直接用于下步反应. 此外, 还发现反应在 白炽灯光照射下进行效果也不明显，这可能与阳光在一 定程度上能催化此类反应进程有关.

在化合物 $8 \mathrm{a} \sim 8 \mathrm{p}$ 合成过程中, 开始用 $\mathrm{ClCH}_{2} \mathrm{CO}_{2}$ $\mathrm{C}_{2} \mathrm{H}_{5}$ 进攻噻唑烷二酮 $\mathrm{N}$ 上的 $\mathrm{H}$, 发现没有产物生成, 接 着我们采用反应活性较好的 $\mathrm{BrCH}_{2} \mathrm{CO}_{2} \mathrm{Bu}-t$ 代替前者, 反应顺利进行，接着用 $\mathrm{CF}_{3} \mathrm{COOH}$ 水解酯键，两步反应 都有较高的产率，而且反应时间短，条件温和，后处理 简单. 


\section{2 生物活性}

目标化合物对金黄色葡萄球菌(S. aureus 4220)、变 形链球菌(S. mutans 3056)、大肠杆菌 (E. coli 1356)的活 性测试结果见表 1 . 对革兰氏阳性菌, 临床上常见的耐 甲氧西林金黄色葡萄球菌(methicillin-resistant S. aureus) 和耐喹诺酮金黄色葡萄球菌 (quinolone-resistant $S$. aureus)活性测试结果见表 2 .

表 1 化合物 $7 \mathbf{a} \sim 7 \mathrm{p}$ 和 $8 \mathrm{a} \sim 8 \mathrm{p}$ 的抗菌活性(最小抑制浓度, $\mu \mathrm{g} / \mathrm{mL}$ )

Table 1 Inhibitory activity (MIC, $\mu \mathrm{g} / \mathrm{mL}$ ) of compounds $7 \mathbf{a} \sim$ $7 p$ and $8 \mathbf{a} \sim 8 p$ against bacteria

\begin{tabular}{cccc}
\hline Compd. & S. aureus 4220 & S. mutans 3056 & E. coli 1356 \\
\hline $\mathbf{7 a} \sim \mathbf{7 p}$ & $>64$ & $>64$ & $>64$ \\
$\mathbf{8 a}$ & $>64$ & $>64$ & $>64$ \\
$\mathbf{8 b}$ & $>64$ & $>64$ & $>64$ \\
$\mathbf{8 c}$ & $>64$ & $>64$ & $>64$ \\
$\mathbf{8 d}$ & $>64$ & $>64$ & $>64$ \\
$\mathbf{8 e}$ & $>64$ & $>64$ & $>64$ \\
$\mathbf{8 f}$ & $>64$ & $>64$ & $>64$ \\
$\mathbf{8 g}$ & 8 & 32 & $>64$ \\
$\mathbf{8 h}$ & $>64$ & $>64$ & $>64$ \\
$\mathbf{8 i}$ & 8 & 32 & $>64$ \\
$\mathbf{8 j}$ & $>64$ & $>64$ & $>64$ \\
$\mathbf{8 k}$ & $>64$ & $>64$ & $>64$ \\
$\mathbf{8 1}$ & 16 & 32 & $>64$ \\
$\mathbf{8 m}$ & 8 & 8 & $>64$ \\
$\mathbf{8 n}$ & $>64$ & $>64$ & $>64$ \\
$\mathbf{8 0}$ & $>64$ & $>64$ & $>64$ \\
$\mathbf{8 p}$ & $>64$ & $>64$ & $>64$ \\
Norfloxacin & 2 & 1 & 16 \\
Oxacillin & 1 & 1 & $>64$ \\
\hline & & &
\end{tabular}

初步活性测试结果表明, 噻唑烷酮二酮 $\mathrm{N}$ 上无乙酸 链取代的中间体 7a 7p 在 $64 \mu \mathrm{g} / \mathrm{mL}$ 浓度下均无抗菌活 性. 化合物 $8 \mathrm{~g}, 8 \mathrm{i}$ 和 $8 \mathrm{~m}$ 抗菌效果最好, 对金黄色葡萄球 菌(S. aureus 4220)的最小抑制浓度达 $8 \mu \mathrm{g} / \mathrm{mL}$, 但仍不 如诺氟沙星和苯唑西林. 同样, $8 \mathrm{~g}, 8 \mathrm{i}$ 和 $8 \mathrm{~m}$ 对变形链球 菌(S. mutans 3056)也表现出一定抑制作用, 与前者相比 活性稍弱，说明金黄色葡萄球菌对此类化合物的敏感性 高于变形链球菌. 另外, 对金黄色葡萄球菌和变形链球 菌活性顺序: $8 \mathrm{~g}>7 \mathrm{~g}, 8 \mathrm{i}>7 \mathrm{i}$ 和 $8 \mathrm{~m}>7 \mathrm{~m}$, 说明噻唑烷酮 二酮 $\mathrm{N}$ 上乙酸链能明显提高抗革兰氏阳性菌的活性(至 少 8 倍). 所有化合物对大肠杆菌 1356(属于革兰氏阴性
表 2 化合物 $8 \mathrm{~g}, 8 \mathrm{i}, 8 \mathrm{I}$ 和 $8 \mathrm{~m}$ 对多重耐药革兰阳性菌的抑制活 性(最小抑菌浓度, $\mu \mathrm{g} / \mathrm{mL})^{a}$

Table 2 Inhibitory activity (MIC, $\mu \mathrm{g} / \mathrm{mL}$ ) of compounds $\mathbf{8 g}, \mathbf{8 i}$, $\mathbf{8 1}$ and $\mathbf{8 m}$ against clinical isolates of multidrug-resistant grampositive strains

\begin{tabular}{cccccc}
\hline \multirow{2}{*}{ Compd. } & \multicolumn{2}{c}{ MRSA } & \multicolumn{2}{c}{ QRSA } \\
\cline { 2 - 3 } \cline { 4 - 5 } $\mathbf{8 g}$ & 3167 & 3506 & 3505 & 3519 \\
$\mathbf{8 i}$ & 4 & 8 & & $>64$ & $>64$ \\
$\mathbf{8 1}$ & 4 & 4 & 8 & 4 \\
$\mathbf{8 m}$ & 4 & 4 & 32 & 16 \\
Norfloxacin & 8 & 4 & 4 & 8 \\
Oxacillin & $>64$ & $>64$ & 1 & $>64$ \\
\hline
\end{tabular}

${ }^{a}$ MRSA 3167, methicillin-resistant $S$. aureus CCARM 3167; MRSA 3506, methicillin-resistant $S$. aureus CCARM 3506; QRSA 3505, quinolone-resistant S. aureus CCARM 3505; QRSA 3519, quinolone-resistant $S$. aureus CCARM 3519.

菌)最小抑制浓度均大于 $64 \mu \mathrm{g} / \mathrm{mL}$.

对多重耐药菌测试结果显示, 所测的 4 个化合物抗 耐甲氧西林金黄色葡萄球菌的最小抑制浓度达到 4 $\mu \mathrm{g} / \mathrm{mL}$ ，活性与诺氟沙星相当，至少 16 倍优于苯唑西林; 化合物 $8 \mathrm{i}$ 和 $8 \mathrm{~m}$ 在抗耐喹诺酮金黄色葡萄球菌的活性方 面 $(\mathrm{MIC}=4 \sim 8 \mu \mathrm{g} / \mathrm{mL})$, 弱于苯唑西林 $(\mathrm{MIC}=1 \mu \mathrm{g} / \mathrm{mL})$. 另外发现，化合物 $8 \mathrm{~g}$ 和 81 对耐甲氧西林金黄色葡萄球 菌的活性要明显优于耐喹诺酮金黄色葡萄球菌; 而 $8 \mathrm{i}$ 和 $8 m$ 对两种多重耐药革兰阳性菌株的抑制活性无明显差 异.

\section{References}

[1] Yuan, Y.; Zhou, C.-H.; Liu, Q.; Song, C.-Z. Chin. J. New Drugs 2007, 16, 343 (in Chinese).

(袁勇, 周成合, 刘嫱, 宋春泽, 中国新药杂志, 2007, 16, 343.)

[2] Ghosh, A.; Mandal, S.; Banerji, A.; Kar, M.; Banerji, J. Nat. Prod. Commun. 2009, 4, 209.

[3] López, S. N.; Castelli, M. V.; Zacchino, S. A.; Domínguez, J. N.; Lobo, G.; Charris-Charris, J.; Cortés, J. C.; Ribas, J. C.; Devia, C.; Rodríguez, A. M.; Enriz, R. D. Bioorg. Med. Chem. 2001, 9, 1999.

[4] Liu, T.; Hu, Y.-Z. Chin. J. Org. Chem. 2006, 26, 983 (in Chinese). (刘滔，胡永洲，有机化学, 2006, 26,983.)

[5] Bekhit, A. A.; Habib, N. S.; Bekhit, A. Boll. Chim. Farm. 2001, 140, 297.

[6] Bhattarai, B. R.; Kafle, B.; Hwang, J. S.; Khadka, D.; Lee, S. M.; Kang, J. S.; Ham, S. W.; Han, I. O.; Park, H.; Cho, H. Bioorg. Med. Chem. Lett. 2009, 19, 6161.

[7] Patil, V.; Tilekar, K.; Mehendale-Munj, S.; Mohan, R.; Ramaa, C. S. Eur. J. Med. Chem. 2010, 49, 4539.

[8] Liu, X.-F.; Zheng, C.-J.; Sun, L.-P.; Liu, X.-K.; Piao, H.-R. Eur. J. Med. Chem. 2011, 46, 3469.

[9] Jung, S. H.; Park, S. Y.; Kim-PAK, Y.; Lim, S. S. Chem. Pharm. Bull. 2006, 54, 368.

[10] Giles, R. G.; Lewis, N. J.; Quick, J. K.; Sasse, M. J. Tetrahedron 2000, 56, 4531.

[11] Clinical and Laboratory Standards Institute Methods for Dilution Antimicrobial Susceptibility Tests for Bacteria that Grow Aerobically, Approved Standard M7-A6, Clinical and Laboratory Stan- 
dards Institute, Wayne, PA, USA, 2003

[12] (a) Norikane, Y.; Itoh, H.; Arai, T. J. Phys. Chem. A 2002, 106, 2766 . (b) Zhao, P.-L.; Wang, F.; Huang, W.; Chen, Q.; Liu, Z.-M. J. Org. Chem. 2010, 30, 1567 (in Chinese).

(赵培亮, 王福, 黄伟, 陈琼, 刘祖明, 有机化学, 2010, 30, 1567.)

(Lu, Y.; Fan, Y.) 